\title{
Implementation of Resilience Engineering for Thoracic Surgery. Reconciling Work-as-Imagined and Work-as-Done
}

\author{
Takanori Ayabe1,2*, Masaki Tomita1, Ryo Maeda1, Manabu Okumura², Kunihide Nakamura ${ }^{2,3}$, \\ Kazue Nakajima ${ }^{4}$ \\ ${ }^{1}$ Division of Thoracic and Breast Surgery, Department of Surgery, Faculty of Medicine, University of Miyazaki, Miyazaki, Japan \\ ${ }^{2}$ Department of Patient Safety Management, University of Miyazaki Hospital, Miyazaki, Japan \\ ${ }^{3}$ Division of Cardiovascular Surgery, Department of Surgery, Faculty of Medicine, University of Miyazaki, Miyazaki, Japan \\ ${ }^{4}$ Department of Clinical Quality Management, Osaka University Hospital, Osaka, Japan \\ Email: ^tayabe@med.miyazaki-u.ac.jp
}

How to cite this paper: Ayabe, T., Tomita, M., Maeda, R., Okumura, M., Nakamura, K. and Nakajima, K. (2020) Implementation of Resilience Engineering for Thoracic Surgery. Reconciling Work-as-Imagined and Work-as-Done. Surgical Science, 11, 257-273. https://doi.org/10.4236/ss.2020.119028

Received: August 7, 2020

Accepted: September 25, 2020

Published: September 28, 2020

Copyright $\odot 2020$ by author(s) and Scientific Research Publishing Inc. This work is licensed under the Creative Commons Attribution International License (CC BY 4.0).

http://creativecommons.org/licenses/by/4.0/ (c) (i) Open Access

\begin{abstract}
Background: The Work-As-Imagined (WAI) is a plan that is expected to be performed before surgery, and the Work-As-Done (WAD) is the result of work actually done. In order to perform safe and high-quality surgery for the individual surgeon, the surgical team, and hospital organization as a system, we have to reconcile the WAI and the WAD in resilience engineering for the real world of surgical healthcare. Objective: Based on the resilient healthcare theory, we would like to clarify the actual way of reconciling the WAI and WAD in surgery. Material and Methods: As a typical model of thoracic surgery, we use a lobectomy case for lung cancer. We describe a surgeon's WAI and WAD, and we explain the anticipating, monitoring, responding, and learning based on the resilient healthcare theory. We reveal the gaps between the WAI and WAD during an operation, we consider the surgeon's thinking and actual performance, and we describe the actual way of reconciling the WAI and WAD for the surgeon and surgical team. Outcomes: We described three scenes in the operating room, which are 1) by individual surgeon: adjustment for intrathoracic adhesion; 2) by surgical team: adjustment for pulmonary artery bleeding; and 3) by surgical team with multi-professionals in the operating room: adjustment for life threatening pulmonary artery critical bleeding. Conclusion: In order to implement a resilient healthcare theory in everyday surgical work, it is important that 1) learning of incidents and the experience of doing well for unexpected events as lessons, and 2) constructing a circulation mechanism of anticipating, monitoring, responding, and learning.
\end{abstract}




\section{Keywords}

Work-as-Imagined (WAI), Work-as-Done (WAD), Surgery, Resilience Engineering

\section{Introduction}

Surgical treatment is a personalized treatment tailored to the patients. Based on a preoperative medical examination, the preoperative diagnostic imaging of a patient is presented at the surgery conference, which is multifaceted as considered by the surgical team. The preoperative health condition of the patient is discussed regarding the severity of the underlying disease, the presence or absence of preoperative comorbidity, age, daily activity, and envisaged risk. In case of lung cancer surgery, based on the clinical stage of the cancer, the balance of the operative benefit and harm, and by estimation of the radicality of cancer and the degree of the expected postoperative complications, an optimal operative plan is considered.

Based on the resilience engineering [1] [2] [3] [4], the preoperative surgical simulation which a surgeon considered and planned is the Work-As-Imagined (WAI). Scheduled surgery and the procedure are explained to the patient, and informed consent obtained, thus the so-called preoperative WAI is determined. The preoperative WAI contains the flow scheduled during the preoperative examination, surgical plan, and postoperative complications management. Surgery is based on the technical skill such as the surgeon's knowledge and the operating technique. By anticipating the assumed and the unexpected events, according to the surgery progress and intraoperative findings, the surgical procedure is changed on a case-by-case basis, and adjusted to be completed. The surgical process of the correspondence and the result are not one and the same surgery.

In contrast, the actual work done during surgery, procedure, progression, and outcomes are Work-As-Done (WAD). Since the surgery in progress involves risk, when we run the WAI, foresee the unexpected and crisis situations, so as not to produce bad results, for safety, the preoperative WAI, it is determined that the impossible such as changing, which is changed sometimes when that must be adjusted. When we faithfully execute the WAI for the unexpected, because it may become a crisis situation, thus to foresee this, it must survive in a realistic WAD. Even if the preoperative WAI greatly deviates from the realistic $\mathrm{WAD}$, in fact, as a result of adjusting the preoperative WAI, so that we are realizing the WAD. For an unscheduled surgery WAD due to the occurrence of unexpected events, it is against the crisis bleeding or to cope with another surgical procedure type WAD. Due to the corresponding WAD in resilience, even though far from the preoperative WAI, it is a successful WAD.

The surgeon, actually or WAD is the one different from the same that went with "surgery WAI" to think in the head before surgery, continue to progress 
repeatedly experience. As an example case of lung cancer surgery, we will explain the concept of the WAI and WAD with adjustment cases.

In the present study, in order to reveal the actual way to reconcile the WAI and $\mathrm{WAD}$, that is, feedback with an intraoperative video, to observe the level of surgeons, teams, and system perspectives.

\section{Materials and Methods}

\subsection{Decision Process of Preoperative WAI}

Figure 1 shows the flow of the preoperative decision process of the WAI for the surgical treatment of lung cancer. The treatment of lung cancer, and the guidelines of clinical reference, the condition of the patient, the clinical tumor stage, surgery, chemotherapy, and this in combination with radiation therapy.

As a guideline of NCCN [5], we consider the latest evidence obtained from clinical research, which has created the guidelines listed for the recommended treatment methods and selection. The guidelines show a standard treatment outline for lung cancer diagnosis (academic WAI). Patients screened for lung cancer from a discovered abnormal chest shadow as was suspected, a precision inspection, such as a bronchoscopy, is carried out. Based on the practice guidelines, the recommended treatment plan is determined and explained to the patient.

In the case of a relatively early stage and resectable lung cancer, surgical resection is the first choice. Advanced lung cancer and unresectable lung cancer are treated with chemotherapy and radiation other than surgery. The patient is introduced to the surgical team with physicians from internal medicine, and lung cancer is performed to consider whether it is possible to resect, whether it is possible for general anesthesia, the presence and extent of comorbidities and

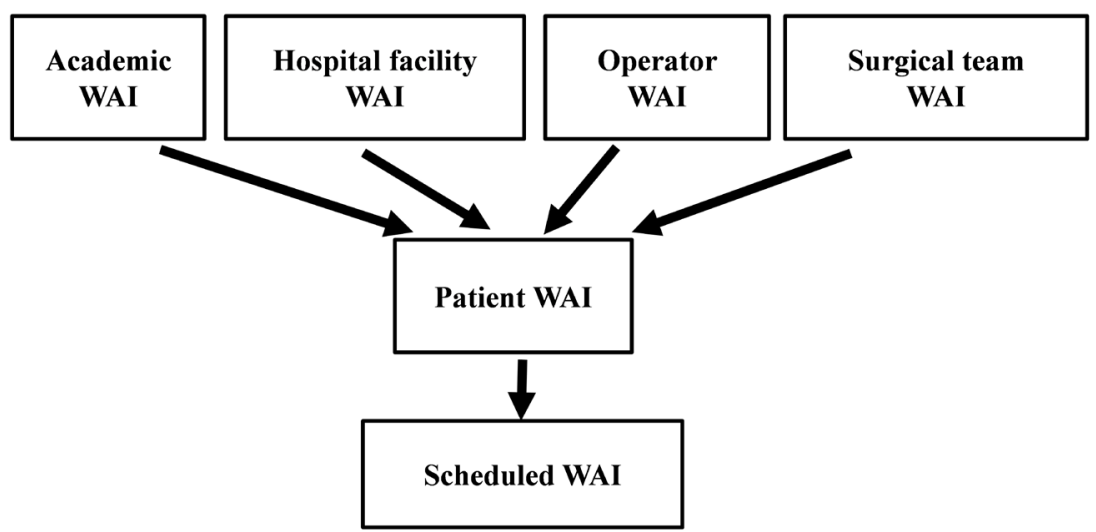

Figure 1. "Academic WAI" is based on the guideline for treatment by an academic society. "Hospital facility WAI" is based on the guideline for treatment by a hospital facility. "Operator WAI" is planned by the individual surgeon's knowledge, technique and experience. "Surgical team WAI" is a consensus based on a surgeon's conference. "Patient WAI" is based on a patient's background and characteristics. In the case of the preoperative WAI for the surgical treatment of lung cancer, there are many WAIs which means academic, hospital facility, operator, surgical team, and patient WAI. The figure shows a flow chart and we finally determine the scheduled WAI by reconciling those WAIs. 
complications other than lung cancer are evaluated. Complications that can occur after surgery, outcomes and risks of the planned surgery are considered and studied. Through the preoperative review process, the WAI will be determined (hospital facility WAI and surgical team WAI). Based on the WAI, the surgeon's plans are described to a patient, consent is obtained, and the final surgical WAI is determined (operator WAI).

The treatment of rare cases that do not appear as difficult cases or guidelines that lead to the selection of treatment methods, conferences in a system called team medicine by specialists in respiratory medicine, radiology and chest surgery, computed-tomography (CT), magnetic resonance imaging (MRI), the imaging findings and literature, such as positron emission tomography-computed tomography (PET-CT), are considered for the multifaceted case to determine the best method of treatment. Based on the guidelines the treatment plan WAI of the individual patient is determined, the surgical technique is discussed in the surgical conference, and preoperative WAI (surgical team WAI) is determined.

However, based on the patient factors, such as a patient's hope, the WAI, from the surgical procedure is a standard one, it may become an aggressive expansion surgery and passive reduction surgery (patients WAI).

The patient hears a description of the surgery, understands and consents to, thus agreement on the scheduled surgery is determined as the final preoperative WAI (scheduled WAI).

\subsection{Procedure and Flow of Surgery (Lobectomy for Lung Cancer)}

As a typical model of thoracic surgery, we explain the case of a lobectomy for lung cancer. Figure 2 shows the process of standard lung cancer surgery such as a right upper lobectomy procedure (WAI). In the case of the right upper lobe lung cancer, the right upper lobe must be excised. Patients are in the lateral decubitus position, the chest skin is cut $(10-15 \mathrm{~cm})$, and a thoracotomy at the 5th intercostal space reaching into the thoracic cavity. The patient is ventilated only on the left lung and collapsed on the right puffy lung to make the lung smaller, then surgery is performed. The right lung is divided into three parts; i.e., the upper, middle and lower lobes. The upper, middle and lower lobes are divided, and the pulmonary artery going to the upper lobe and the blood vessels of the pulmonary veins are detached, confirmed, exposed, and safely separated so as not to bleed from the blood vessels. After processing the blood vessels, the upper lobe bronchus is cut off to terminate the upper lobe resection. The mediastinal lymph nodes are then dissected, and the standard procedure for lung cancer, i.e., the upper lobectomy with mediastinal lymph node dissection is completed. We check the bleeding and stop any bleeding before closing the chest. If there is air leakage from the lungs, a suture closure procedure is performed. A chest drain is inserted, the open chest is closed, the skin is sutured closed and the surgery is finished. This is textbook surgery and the WAI for the surgeon preoperatively assumed comes to mind. 


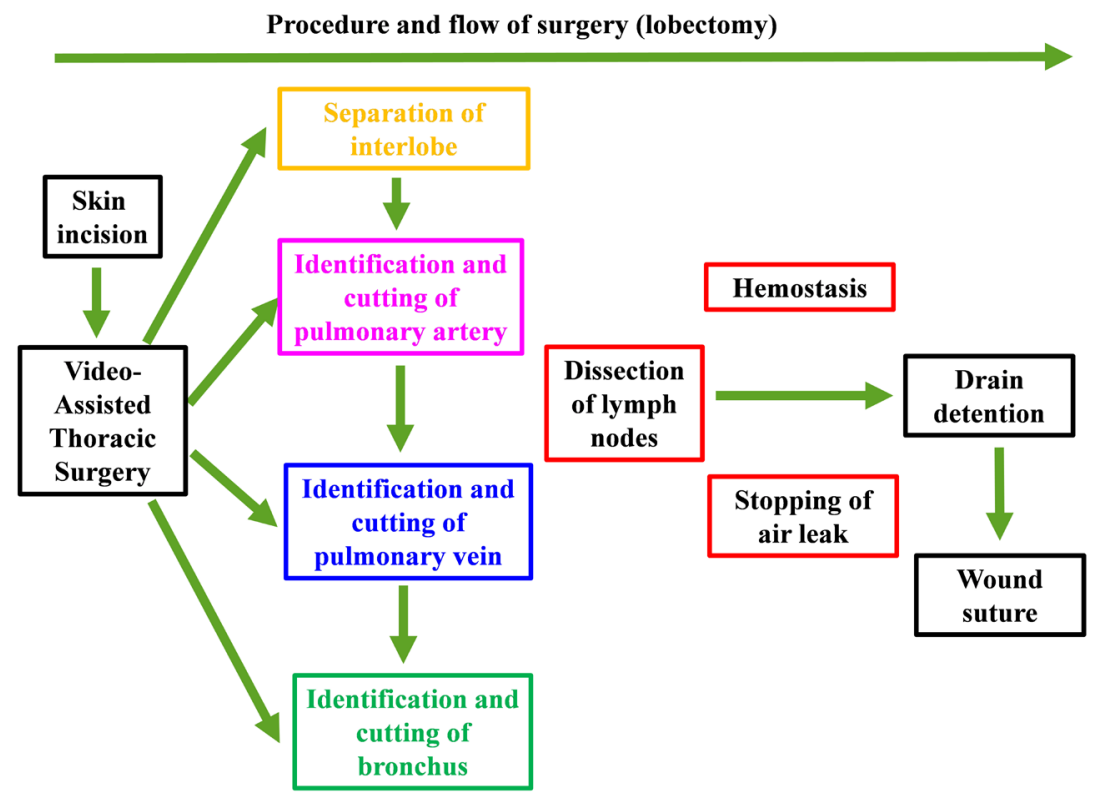

Figure 2. Flow chart shows a surgical standard procedure regarding the lobectomy for lung cancer, and this is the so-called surgical WAI. In case of a right upper lobectomy, after skin incision of 3 to 4 holes, a videoscope is inserted in the thorax, and video-assisted thoracic surgery is performed with endoscopic forceps. A surgeon separates the interlobes of the upper, middle, lower lobes and identifies the interlobular pulmonary artery with branches and pulmonary veins and cuts these vessels with an automatic suturing device. A surgeon identifies the upper bronchus and cuts the bronchus with an automatic suturing device. After the upper lobectomy, the mediastinal lymph nodes are dissected. After hemostasis and stopping of any air leaks from the lung, a chest drain is installed. After wound closure, the surgical procedure is finished. As the usual surgical flow, this figure displays the process of lung resection during surgery.

\section{Results}

We used a model case of a lobectomy for lung cancer (Figure 2) and we considered the gaps between the WAI (preoperative plan) and the WAD (performed surgery). We revealed the work of anticipating, monitoring, adjustment, and responding in resilience engineering. Based on the standard flow of the lung cancer operation (Figure 2), we described three scenes of adjusted examples (Figure 3, Figure 4, and Figure 5) in the surgeon's actual adjustment in the operation. The three responding and adjustment patterns that are often seen are 1) intrathoracic adhesion in everyday normal time, 2) rare moderate bleeding events, and 3) expanded massive hemorrhage such as critical life-threatening harm event in the unexpected emergency situation within the expected range.

\subsection{Everyday Normal Time by Individual Surgeon: Monitoring, Responding, and Adjustment of Intrathoracic Adhesion}

Figure 3 shows the case of the actual response and adjustment to the unexpected intrapleural pleurodesis in thoracic surgery. During the video-assisted thoracic surgery, adhesions are found throughout the intrathoracic, which has not been assumed in the initial WAI. Figure 3 shows the additional procedure 


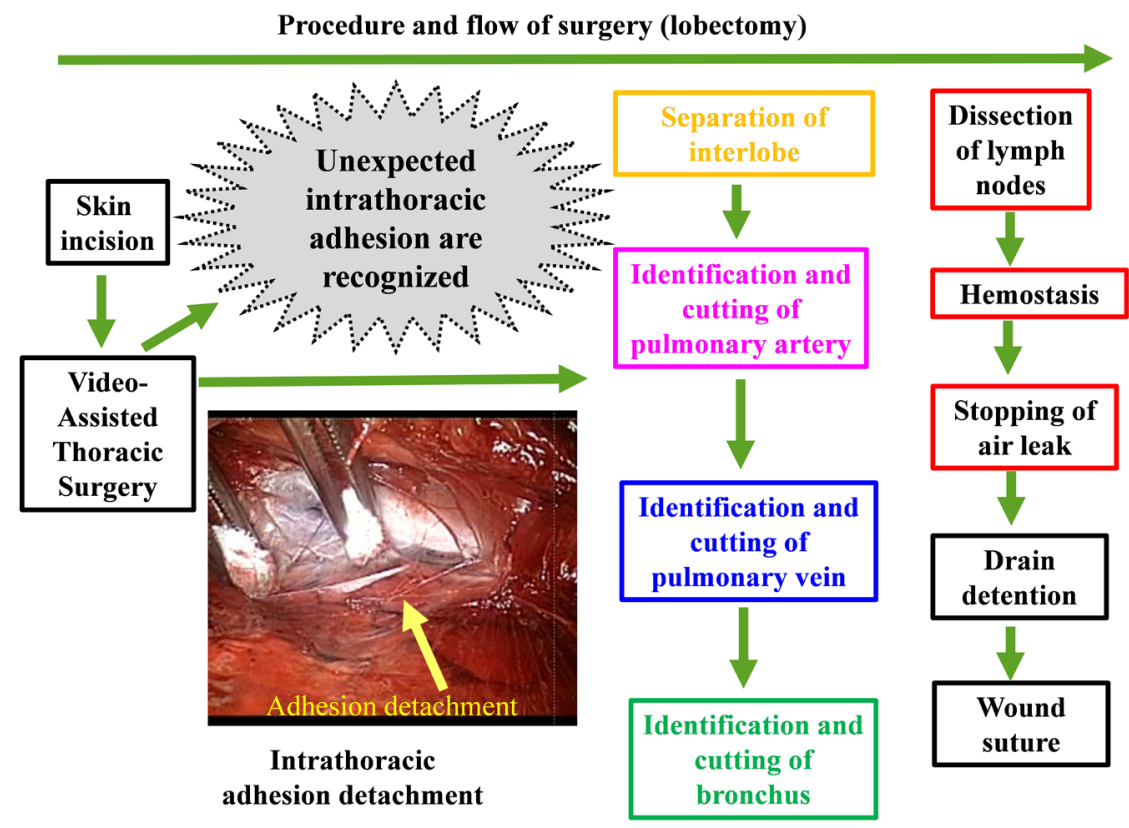

Figure 3. If an unexpected intrathoracic adhesion is recognized during the operation, this figure shows changes in the surgical flow, that is, adding an extra procedure to the whole process, which is an adjustment by responding for the adhesion detachment, while the other process has not been changed, thus it may take a long time to deal with it. Based on the resilient healthcare theory, this adjustment is within the everyday normal procedure by the individual surgeon, that is, monitoring of the intrathoracic adhesion and responding by adhesion detachment.

of responding and adjustments to the unexpected intrathoracic pleurodesis (as an example of reconciling the WAI and WAD).

The adhesions must be exfoliated to be ready for lung resection of the WAI. If we do not exit the adhesiolysis, we cannot proceed with the lobectomy. Furthermore, since the lungs are adhesions, during the operative course of adhesiolysis, as lung damage, we foresee that the bleeding and air leakage from the site of injury is sustained, and carefully peeled off. If the lung has much damages, suture hemostasis and suture closure occurs, surgery time is prolonged, a large amount of bleeding happens, surgical stress increases, a blood transfusion is required, and the WAD is far from the initial scheduled WAI. Thus, it happens that unexpected events occur within the expected range, thus it must be correspondingly adjusted.

\subsection{Everyday Normal Time by Surgical Team: Monitoring, Responding, and Adjustment of the Pulmonary Artery Bleeding}

Figure 4 shows the case of an actual response and adjustment to an unexpected pulmonary arterial hemorrhage due to vessel injury during the peeling and exposure process of the vessel. There are expected pulmonary adhesions due to chemotherapy for cancer invasion, showing the procedure of adjusting the corresponding unexpected bleeding during the peeling process of the pulmonary artery adhesions. There is the natural stop levels of small bleeding due to the 


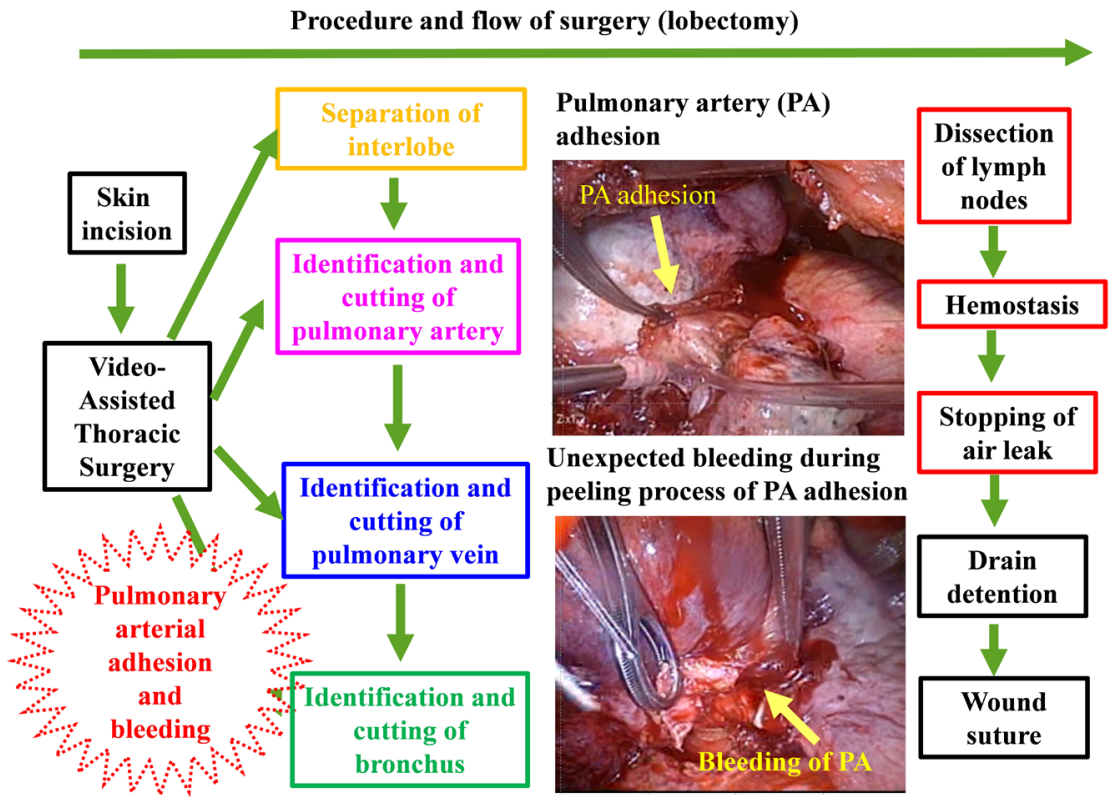

Figure 4. If the adhesion of interlobular pulmonary artery due to chemotherapy is recognized during the peeling process of the pulmonary artery, this figure shows a partly changed process, that is, adding an extra procedure to the whole process, which is an adjustment by responding to the pulmonary arterial bleeding and stopping the hemorrhage, while the other process has not been changed, thus it may take some time to deal with it. Based on the resilient healthcare theory, this adjustment is within the everyday normal procedure by the surgical team, that is, monitoring of the pulmonary arterial adhesion and the bleeding, and responding to the bleeding, and adjustment by stopping the pulmonary artery bleeding.

Gauze pressure and also the levels of hemostasis with hemostatic and the direct suturing of the vascular lesion that does not stop only by compression. There is also a level of major hemorrhage that causes an injury site to expand and become a large blood vessel injury leading to a life crisis.

In the case of small bleeding, we have to adjust by the WAD such as compression hemostasis with operation gauze. If we do not have complete hemostasis, we have to adjust in hemostasis operation by vascular suture hemostasis, without significantly affecting the overall operation progress. However, in the case of moderate or severe bleeding, for example, in addition to another unexpected and expanded bleeding, that is, it failed the initial WAD, the vascular injury is likely to develop into the next crisis. In the case of the migration to critical bleeding status, we have to foresee a new risk of the next massive bleeding, and we have to adjust the crisis by the next new WAD of a reliable hemostasis operation.

\subsection{Unexpected Emergency Situation (Crisis State Such as Intraoperative Massive Bleeding) by Surgical Team with Multi-Professionals in Operating Room: Monitoring, Responding, and Adjustment of the Massive Hemorrhage}

Figure 5 shows the cooperative crisis management flow for a life-threatening case due to critical massive bleeding and shows the procedure (WAI and WAD) 


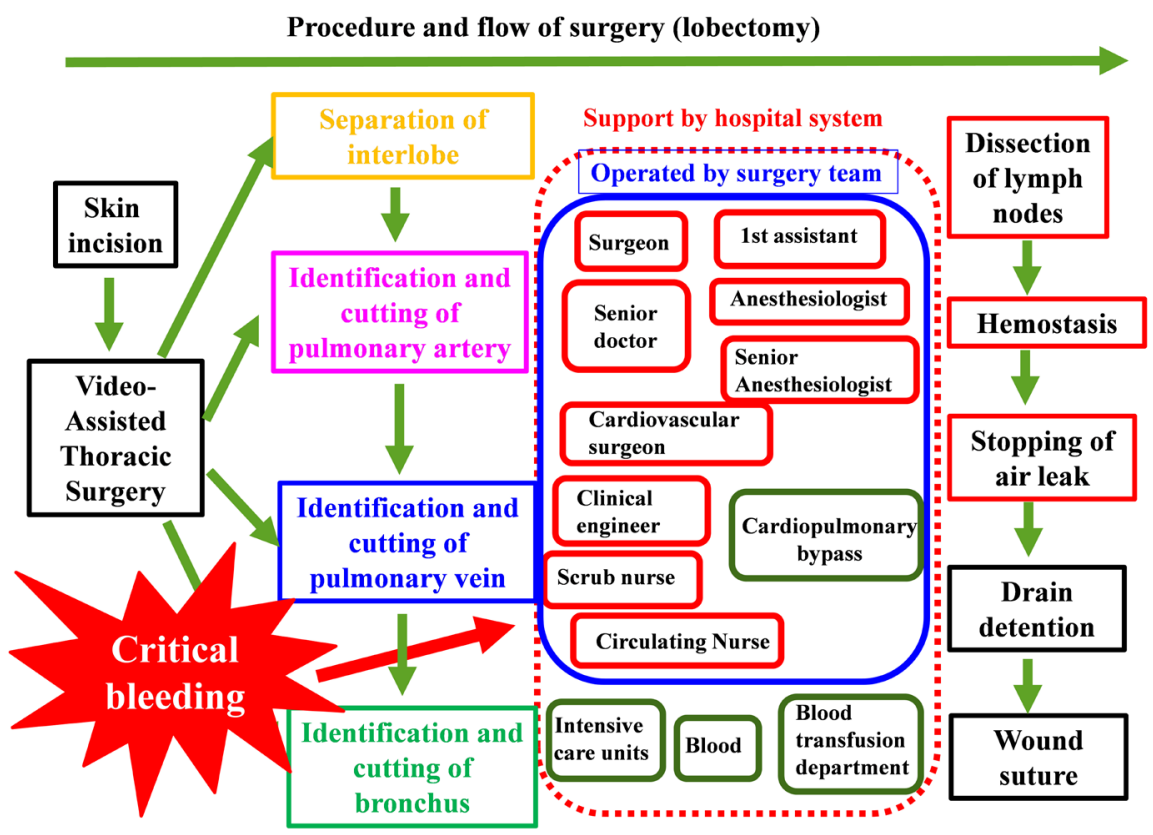

Figure 5. If massive bleeding of the pulmonary artery is recognized due to expanded injury of the vessel and critical bleeding during the hemostasis process of pulmonary arterial bleeding, this figure shows a significantly changed process, that is, changed to an extra and another procedure, which is an adjustment by responding to the pulmonary arterial bleeding and stopping the massive hemorrhage, and saving the patient's life, thus a new surgical team with a cardiovascular surgeon is started and supported by the hospital system with multi-professionals. Based on the resilient healthcare theory, this adjustment is within the unexpected emergency situation (crisis state such as intraoperative massive bleeding) by the surgical team with multi-professionals in the operating room, that is, monitoring of the crisis, responding to life threatening event, and adjustment of the massive hemorrhage and saving the patient's life.

for dealing with and adjusting for unforeseen large amounts of bleeding and critical massive bleeding leading to a life crisis. We have to respond to the lifethreatening status with resilience and practice the WAD that deviates from the WAI. With the corresponding adjustment to the high amount of unexpected bleeding, the WAD is beyond the technical skill of the surgeon personal hemostasis operation.

In the WAD of the surgical team, the operator has to work to be compatible with the cooperation of the anesthesiologist and senior doctors. In addition to the WAD under a crisis situation, operative personnel such as cardiovascular surgeons, clinical engineers, laboratory technician in the blood transfusion unit, and medical staff in the intensive care unit, recognizes that the degree of influence is expanding. In the actual WAD, not only the support of the cardiovascular surgeon, clinical engineers manipulate the artificial heart-lung pump, thus the goal of the operation has changed to be far greater from the scheduled surgery formula of the first lung cancer removal (WAI), thus the corresponding $\mathrm{WAD}$ is resilient, emergency hemostasis and lifesaving.

With the participation of the operator, anesthesiologist, cardiovascular surgeon, 
technician in the blood transfusion unit, clinical engineers, circulating nurses, and medical staff involving the intensive care unit, as a system of hospital organization, a cooperation hospital team with a life-saving purpose are established and should be functioning. With massive bleeding, unexpectedly, it will have a bad effect on the flow of the surgery, disturbance, and emergency situation. It becomes a crisis situation for these emergency situations to correspond with resilience.

The WAI for hemorrhage constantly monitors any unexpected hemorrhage during the operation, recognizes blood vessel status, correctly identifies the hemorrhage site in the case of injury, determines the amount of hemorrhage, and assumes normal vision to ensure a sufficient visual field from the WAI for bleeding. That is, for the actual WAD to bleeding events, bleeding stops with pressure hemostatic gauze, when we did not stop to think about what to do, foresee the worst-case scenario, life to determine the boundaries of the crisis situation, preparation of blood transfusion, determine the amount of bleeding, or to maintain the vitals of the patient, to reconfigure the surgical team, corresponding with the entire hospital system, to adjust, and it is essential to continue to cope with resilience.

For the WAD of the actual unexpected bleeding, the bleeding site is squeezed for between 10 to 20 minutes, if hemostasis, since it is time margin, the following $\mathrm{WAD}$ is to determine suturing or reinforcing the bleeding site. If bleeding, and only pressure hemostasis cannot cope, it is determined that there is no time to spare, while the pressure hemostasis occurs again, consider the alternative of the hemostasis method WAD, i.e., preparation, must be performed.

For critical hemorrhage, WAI is a new operation with the cooperation of a surgeon, a first assistant, a second assistant, and a surgical team working together with a senior surgeon, and with the cooperation of a cardiovascular surgeon who is used to hemostasis and reorganize the surgical team, anesthesiologist with the cooperation of multi-professionals, such as the hospital system, circulating nurse, anesthesiologist, clinical engineers, technician in blood transfusion unit, an intensive care unit physician, the hospital system united, that is resilient in a crisis, that is assumed at the normal time.

In practice, the surgeon's WAD has to specialize in compression and hemostasis to stop the bleeding. The anesthesiologist's WAD prepares for blood transfusion, performs the blood transfusion, determines the amount of blood loss, vital maintenance of the patients, vital monitoring with multiple persons, and copes with them so as not to allow hypoxia. The nurse's WAD in the surgical field involves preparation of surgical instruments for performing the gauze count, accurate grasp of the amount of bleeding, and hemostasis that corresponds to the instrument count. The WAD of the circulating nurse involves the drip line to check and provide accurate enforcement of the blood transfusion.

With respect to the maximum of the critical situation, the WAD of the multidisciplinary team, each makes their own professional WAD, in cooperation with 
people of the other multidisciplines, and perform the WAD as a strong professional risk group. In normal times, it is assumed the WAI of the critical emergency situation corresponds with the WAD of resilience and supports it. In the event of a serious emergency, depending on the resilience response, the WAD will be far from the initially envisioned WAI, and as a result, even if the preoperative WAI and WAD are different, it is only necessary to save the patient's life. Even if the WAD is far from the original WAI, it is a successful WAD.

\section{Discussion}

\subsection{The Method of Reconciling the WAI and WAD}

Figure 6 shows the concept of bringing the WAI closer to the WAD. We have to compare the initial WAI and the practical WAD. We have to create a new reconstructed WAI as a modified WAI and try to perform the WAD based on the modified WAI.

The surgery plan (WAI) is scheduled as the "surgery WAI" from the preoperative examination, and we perform the actual surgery, which is the so-called WAD. We look back on these performed surgical processes from the postoperative view point. By repeatedly experiencing this review process, the preoperative WAI (surgery plan) and the postoperative WAD get closer. It is important to go through this cycle. After we experienced the preoperative WAI and the intraoperative WAD, we review the preoperative scheduled WAI and the actual performed WAD obtained during the operation, and compare the differences and gaps between the WAI and the WAD in the real world of operation, and from the actual surgical WAD performed, the scheduled "surgery WAI" for the next case will be reviewed.

\section{Reconciling WAI and WAD}

(1) Setting WAI and performing WAD

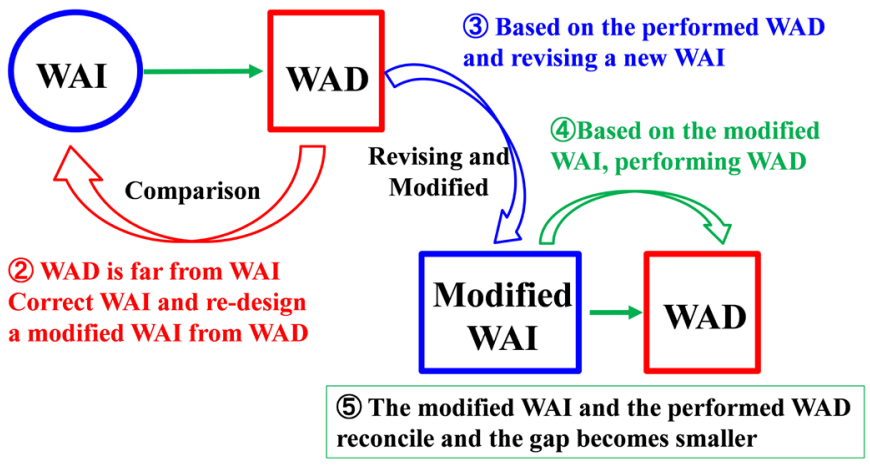

Figure 6. Figure shows a method of reconciling the WAI and WAD. After comparison of the WAI and WAD, modification of the WAI is considered from the performed WAD. If the performed WAD is far from the initial WAI, the next new WAI should be corrected and redesigned as a next modified WAI. Based on the performed WAD, the new corrected WAI is revised. Based on the new modified WAI, the next new WAD is performed. By repeating this cycle of comparison, rebuilding and modification, the modified WAI and the performed WAD gets closer and the gap becomes smaller. 
In addition, when setting the preoperative WAI for the next expected case, the past experience of the intraoperative WAD of the previous operation is helpful. If the difference and gaps between the WAI and WAD are significant, the previously set WAI is corrected (modified WAI), and this becomes the new WAI. Also, this newly modified WAI setting is actually tried in the next operation, and the WAD is actually performed based on the newly modified WAI that should approach the modified WAI. For the revised new WAI and the actual WAD, the gaps between the WAI and the WAD are reviewed and compared, and the distance is further corrected. Next, the newly made WAI is reviewed and a more accurate WAI is created.

By successfully performing these steps and cycles, the preoperative WAI and the actual WAD become similar as much as possible. By modifying the preoperative WAI based on the actual WAD, the WAI can be brought closer to the $\mathrm{WAD}$, and it is important to establish this circulation mechanism.

For young surgeons and practitioners, this cycle has not yet been established. We surgeons learn the surgical procedure manual and textbook before surgery, we put the anatomical knowledge in our head, and participate in the actual surgery assuming the preoperative WAI.

For the actual surgery, the senior surgeon and surgeon underwent a WAD to confirm the experience, aware that the actual WAD and WAI that he has assumed is far greater. It involves how to expose the pulmonary artery and pulmonary vein, from a large number of surgical instruments by selecting any forceps, either of the choices, the operation method, contrivances, learn tips from experience, a WAD that actually works, and a WAD that does not work. They see, touch, experience, remember, forget once, experience, remember, reproduce, thus they have many of these WAD experience memory lessons (repertoire). Many WAD lessons are prepared, and according to the situation, based on a combination of several WAD repertoires, the next WAI is set, monitored, responded, adjusted, and the cycle of performing the WAD is repeated.

In order to bring the $\mathrm{WAI}$ and $\mathrm{WAD}$ closer together, while running this cycle, experiencing success, failure, looking back, learning, successfully turning this circulation mechanism, so that we can improve and naturally, eventually, most of the WAI and WAD gaps will disappear and they will match.

The WAI and WAD to approximate the various lessons, a multi-repertoire is required, in order to remember the lesson at any time, is considered important that must be mastered.

\subsection{Preoperative Surgical Navigation Based on Images of Lung Cancer (WAI)}

As the image support simulation of the WAI, Figure 7 shows the two-dimensional (2D) and three-dimensional (3D) chest computed tomography (CT) images of lung cancer suspected in left lung upper lobe. The left upper lobe has a ground glass shadow suspected of being lung cancer. Preoperatively, it captures the data 


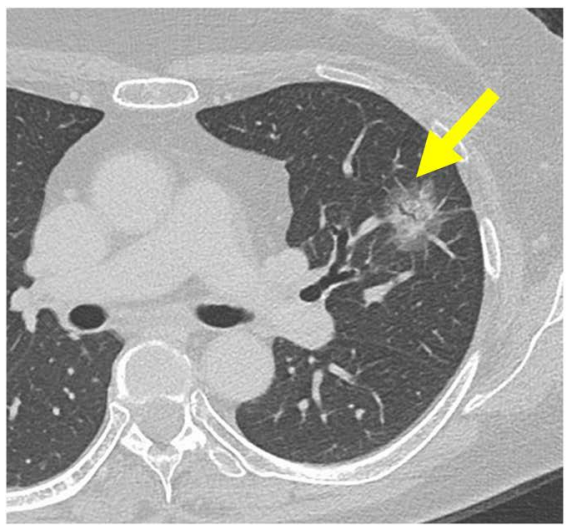

(a)

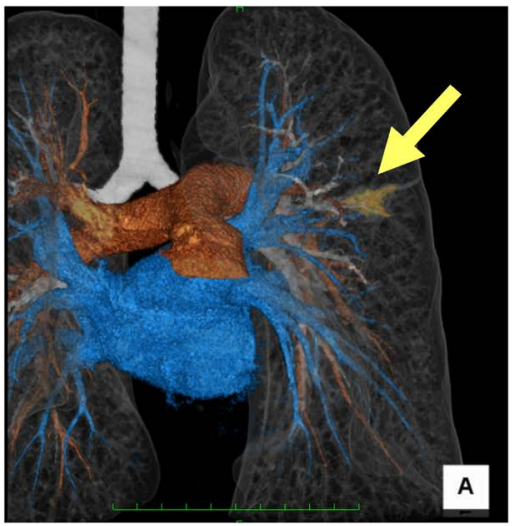

(b)

Figure 7. Preoperative CT images of lung cancer. (a) Two-dimensional CT images. Yellow arrow shows lung cancer shadow; (b) Three-dimensional CT images. Preoperative navigation images. Yellow arrow shows lung cancer shadow.

of the CT picture image on the image support software to build a 3D image. Image support simulation can be moved by the $3 \mathrm{D}$ image is a preoperative WAI, up, down, left and right, free to rotate, confirm the travel of the tumor location and pulmonary artery, thus surgery can be pre-simulation. From the lung organ position of the patient and the positions on the operator's side and the first assistant's side, it is possible to simulate in advance how the pulmonary artery looks three-dimensionally.

\subsection{The Feedback of the Preoperative 3D-CT Images Navigated Simulation (WAI) to the Intraoperative Real Findings (WAD)}

Figure 8 shows the difference and gaps of the preoperative chest 3D-CT images and the intraoperative photo of the pulmonary artery. We specifically introduce how to bring the navigated image of WAI and the real picture of WAD closer together. For example, as shown in Figure 8, the three vascular branches go out the upper lobes from the pulmonary artery, and it can be used for dissecting a branch of the three assumed in the preoperative WAI.

In the actual operation WAD, the same three things (in the case that the WAI is equal to the WAD), or there are four branches as the WAD rather than the three (in the case that the WAI is not equal to the WAD). The state of the operative field can be recorded over a long period of time. After the operation, we can watch the movie and review. Although it takes some time and effort, it is possible to make a short review of the surgical technique in each procedure, create a surgical video, and review. We can review it anytime and anywhere on our computer. It is important to have a process that repeats over and over again whether the preoperative hypothetical WAI that we propose and the WAD we actually experienced are the same or different, approached, or unexpected. Looking back on our surgery third-party in the surgery video is more real when we experience and correct the gap between this pre-planned WAI and the actual WAD and plan our next WAI. 


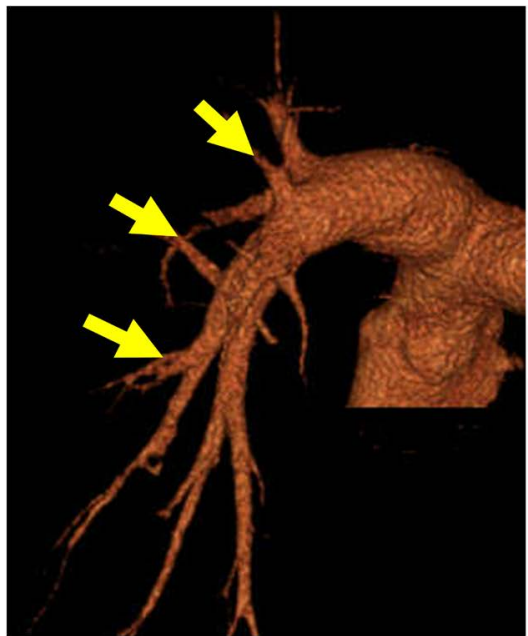

(a)

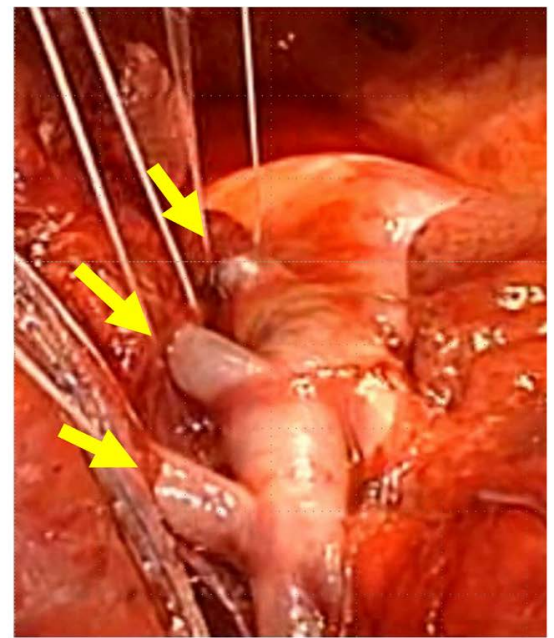

(b)

Figure 8. The comparison and reconciling 3D-CT images of pulmonary artery vessels (WAI) (a) and real vessel branches confirmed during surgery (WAD) (b). (a) 3D-CT images navigated simulation (WAI). Pulmonary arterial branches are preoperatively assumed to be three (three yellow arrows); (b) Intraoperative findings (WAD). Three actual branches are intraoperatively confirmed (three yellow arrows).

It is very useful to set up the WAI with modifications to fit the WAD. By repeating this cycle, the actual WAD approaches the WAI, or the WAD can be realized as expected. As a method of bringing the WAI and WAD closer, both approach each other by bringing the WAI closer to the WAD side or by moving the WAD closer to the WAI side.

\subsection{How to Reduce the Gap between the WAI and WAD}

Figure 9 shows the "surgery WAD" is being recorded by a series of characters, illustration of handwriting, digital photos, and a video. As for the state of the operation, since the WAD, which is an intraoperative finding of the actual operative field from the beginning to the end, is recorded on video, it takes time and effort, but the operation technique can be edited shortly for each point. Surgery of a few hours to 5 to 10 minutes be edited as short as minutes, the surgeon edits its at the point of surgery and in order to look back, it is an important task to look back for learning and education. Surgery video editing also creates an opportunity for a third party to look back on the surgeon's own surgery. Surgery videos are compared with preoperative image findings or recorded as a database.

Surgeons have experienced many gaps between the WAI and WAD. The WAI and the WAD can approach, made in his own surgery a video to review and repeat the self-evaluation. There is also an opportunity to present it at the conference and get opinions from other surgeons. They watch the surgical technique videos of other senior surgeons and learn by comparing them with the surgical videos we performed. We review the success or failure of the surgical technique, examine the situation and reasons, and evaluate it. Of the successful cases and improvement of surgical techniques, such as announcing a video of surgery at an 


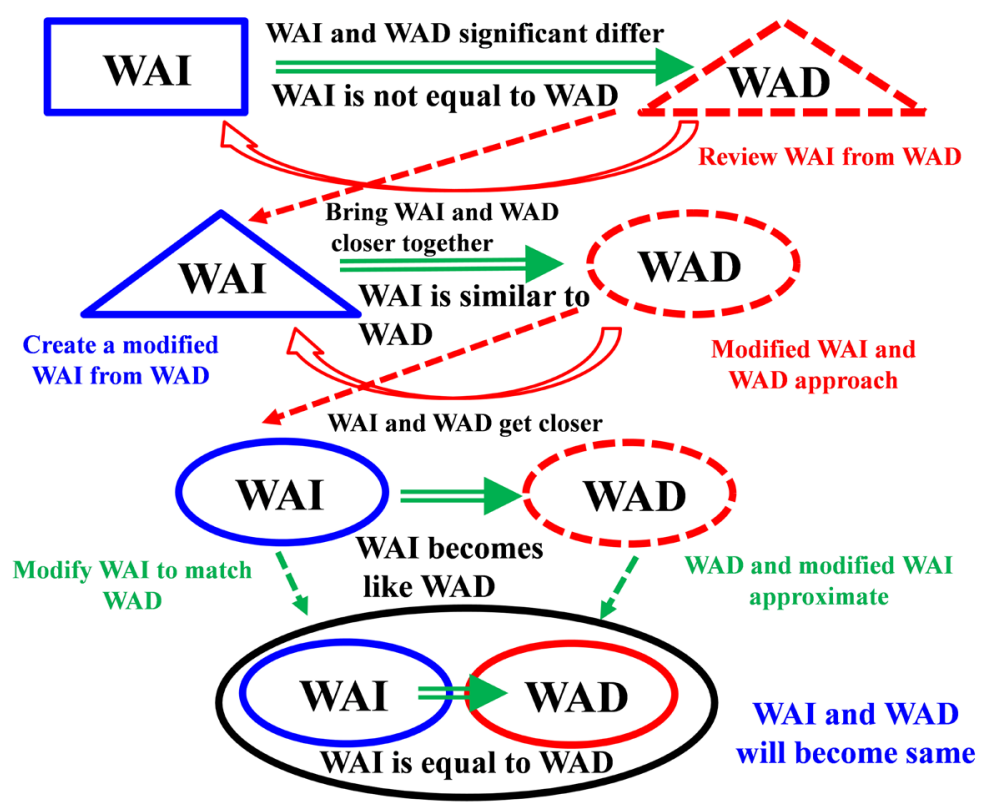

Figure 9. Figure shows how to reduce the gap between the WAI and WAD, and a process of reconciling the WAI and WAD, that is, reviewing, and comparison of WAI and WAD, learning the difference and gaps, and filling the gap between the WAI and WAD. Initially, if the WAI and WAD significant differ, the WAI in not equal to the WAD. After reviewing the initial WAI from the performed WAD, we create a new modified WAI based on the performed WAD to bring the WAI and WAD closer together. At the second time, the WAI becomes similar to the WAD, and the modified WAI and the performed WAD can get closer. At the third time, we modify the WAI to match the WAD, and the modified WAI becomes like WAD, then the WAI and the WAD get together. At the fourth time, the WAD and the modified WAI can approximate. Finally, the WAI is equal to the WAD, and the WAI and the WAD will become the same.

academic meeting, there is also a chance to have them evaluate the surgeons of other facilities. At the Surgical Society Conference, there is a surgical procedure session presentation and the presented video can also be viewed by the surgeons of other facilities. There is also an opportunity to ask questions to the surgeon performing the procedure.

These learning systems has allowed surgeons to discuss and share information about surgical techniques, leading to an improvement in the level of the surgeons as a whole as well as individual surgeons.

\subsection{To Bring the WAI Closer to the WAD, Establishment of a Circular Mechanism to Modify the WAI from the WAD}

One of the important things is to demonstrate resilience in surgery, that is, in order to demonstrate resilience in surgery, it is important to repeat the cycle of 1) anticipating, 2) monitoring, 3) responding, and 4) learning, which is denoted in Figure 10, and integrate technical skills and non-technical skills as well. This is very important. The technical skills of surgery, knowledge and skills in surgery, in particular, the surgical procedure, expansion method, ensure the operative field, recognition of the anatomical positional relationship, hand the exact 
Evaluation of preoperative information and setting of preoperative WAI

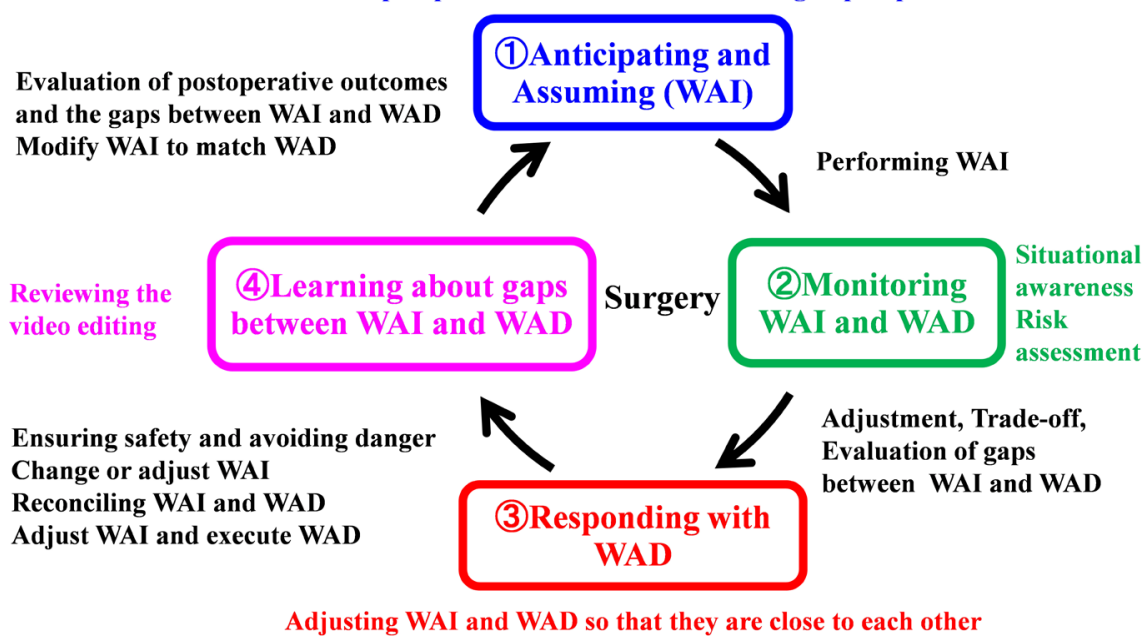

Figure 10. Figure shows a circular mechanism on anticipating, monitoring, responding, and learning in resilient healthcare theory. In order to bring the WAI closer to the WAD, we should establish a circular mechanism to modify the WAI from the WAD. Based on the resilient healthcare theory, the surgeon must be able to perform the operation by anticipating, monitoring, responding, and learning. Based on the evaluation of the preoperative information, we set a preoperative WAI, along with anticipating and assuming adverse events during the surgical progress, the surgeons faithfully execute the WAI (scheduled plan). During the operation, the surgeon monitors the WAI and WAD with situation awareness and risk assessment. For the adverse event within expectations and unexpected events, the surgeon must adjust and respond to them such as adjustment and trade-off, and evaluate the gaps between the scheduled WAI and the actual WAD. The surgeon should respond to the difficulty and selfishness by adjusting the WAI and WAD so that they are close to each other. By ensuring safety, avoiding danger, changing or adjusting the WAI, reconciling the WAI and WAD, and adjusting the WAI and executing the WAD, the surgeon would learn about the gaps between the WAI and WAD. They must review the video editing. By evaluation of the postoperative outcomes and reduction of the gaps between the WAI and WAD, the surgeon can modify the WAI to match the WAD.

type of surgical instruments, then safety measures. There are the ways to avoid dangerous operations.

For the non-technical skills in surgery, situational awareness (information gathering and analysis), decision-making, communication, leadership, and personal factors (stress and fatigue management) are important in the development of surgery.

Resilience skills of surgery, intraoperative trouble of assumptions and predictability within the expected range, ready to correspond to it, situational awareness, tailored to the intra-operative to the ever-changing and intraoperative situation you unexpectedly occur with crisis situation judgment, but the ability to respond by combining the imagination and prediction of the scenario is important.

\subsection{Implementation of Resilience Engineering to Surgery}

Surgery is a live demonstration that reproduces in real time backed by medical 
theory. The flow and know-how for responding to occasions are technique, art, and surgery in response to a variety of patient circumstances (preoperative underlying disease, severity, complications, old age risk, etc.). The initial surgical WAI is set, but the surgical simulation assumed by the surgeon before the operation is the WAI itself. Surgery is a live performance that brings the WAD closer to the WAI. An event that occurs within or beyond the assumption, while the surgeon's confidence is completely self-monitoring and feels that the surgeon is performing well during the operation, while recognizing the surgical findings and the surgical situation. While responding well to the situation, foresee the danger, avoid the danger and proceed. Surgical skills are not enough to make full use of the technical skills called surgical techniques.

In addition to the non-surgical areas and non-technical skills, it is necessary to have the ability to adjust to the occasion and adaptability according to the progress of the surgical procedure. For unexpected events, the planned preoperative WAI is adjusted to the actual WAD in a flexible manner while responding to the resilience. The WAI may be changed or adjusted according to the actual WAD, or the WAI is close to the WAD, so as to approach the WAD to the WAI, make adjustments and be operated in a safe optimum direction for the surgery.

The process of completed surgery is that the actual WAD occurs. Between the WAI and WAD, there are efforts and processes to bring the WAI and WAD closer to each other, and work to fill the gap between the WAI and WAD. The process and the final result considered in the WAI are set, which become the body of the WAD. If the actual WAD is not satisfactory, it is necessary to review and correct the WAI itself from the WAD that did not work. If we are satisfied with the WAD you actually performed, the WAI is a WAI that we can use as is.

If the relationship between the WAI and WAD is good, it evaluates the things that do not work, repeats the gap, modifies it and the WAI and WAD are closer. Whether the WAI is close to the WAD or the WAD is close to WAI, either approach may be used, and the process of bringing the WAI and WAD closer is important.

In order to enhance the resilience ability of the surgery, understanding the surgical procedure WAI itself, standard deployment method, securing the surgical field of view and recognizing the anatomical and spatial positional relationship, learning the correct use of various surgical instruments, selection of appropriate procedures, prevention of safety measures and avoidance of dangerous operations, assumption and preparation of unexpected troubles within the assumed range, recognition of situations that change during the operation, prediction and avoidance of unexpected crisis situations, resilience, perseverance, maintaining a peace of mind, choosing a method to troubleshoot adverse events that occur, acquiring technical and non-technical skills, creating a mood that provides an atmosphere in the right direction, demonstrating leadership, and managing physical condition are important. It is also important to have a lot of fatigue recovery, stress relieving power, and repertoire. 


\section{Conclusion}

In order to implement a resilience engineering in everyday clinical surgical work, it is important that 1) learning of incidents and the experience of doing well for unexpected events, and 2) constructing a circulation mechanism of learning, responding, monitoring, and anticipating.

\section{Acknowledgements}

This study was supported by JSPS KAKENHI Grant Number JP18K04636.

This study was approved by the University of Miyazaki Hospital Clinical Research Ethics Board (No. O-0737) in July 2020.

\section{Conflicts of Interest}

The authors declare no conflicts of interest regarding the publication of this paper.

\section{References}

[1] Hollnagel, E. and Braithwaite, J. (2013) Resilient Health Care (Ashgate Studies in Resilience Engineering).

[2] Braithwaite, J., Wears, R.L. and Hollnagel, E. (2016) Resilient Health Care, Volume 3: Reconciling Work-as-Imagined and Work-as-Done. https://doi.org/10.1201/9781315366838

[3] Wears, R.L. and Hollnagel, E. (2015) Resilient Health Care, Volume 2: The Resilience of Everyday Clinical Work (Ashgate Studies in Resilience Engineering).

[4] Woods, D.D. and Hollnagel, E. (2006) Resilience Engineering: Concepts and Precepts.

[5] NCCN Clinical Practice Guideline in Oncology, Non-Small Cell Lung Cancer. Version 2. 2018. https://www2.tri-kobe.org/nccn/guideline/lung/english/non_small.pdf 